Revue d'histoire de l'Amérique française

WEVUE D.HISTOIRE DE L'AMÉRIQUE FRANÇAISE

\title{
Inventaire critique des notaires royaux des gouvernements de Québec, Montréal et Trois-Rivières (1663-1764) (suite)
}

\section{André Vachon}

Volume 11, numéro 2, septembre 1957

URI : https://id.erudit.org/iderudit/301835ar

DOI : https://doi.org/10.7202/301835ar

Aller au sommaire du numéro

Éditeur(s)

Institut d'histoire de l'Amérique française

ISSN

0035-2357 (imprimé)

1492-1383 (numérique)

Découvrir la revue

Citer cet article

Vachon, A. (1957). Inventaire critique des notaires royaux des gouvernements de Québec, Montréal et Trois-Rivières (1663-1764) (suite). Revue d'histoire de l'Amérique française, 11(2), 270-276. https://doi.org/10.7202/301835ar d'utilisation que vous pouvez consulter en ligne. 


\title{
INVENTAIRE CRITIQUE DES NOTAIRES ROYAUX DES GOUVERNEMENTS DE QUÉBEC, MONTREAAL ET TROIS-RIVIËRES *
}

\author{
(1663-1764)
}

(suite)

PierRe LalanNe

Pierre Lalanne fut nommé par Bigot notaire royal dans l'étendue de la seigneurie de Chambly seulement, le 19 janvier $1752 .{ }^{82}$

Le 19 mars 1753, l'intendant Bigot accordait une autre commission à Lalanne pour exercer également dans la seigneurie de Laprairie de la Madeleine. ${ }^{83}$

Lors du renouvellement des commissions, sous le régime militaire, Gage ne permit à Lalanne d'exercer que dans la seigneurie de Laprairie de la Madeleine, et à la charge d'y demeurer. ${ }^{84}$ C'est le seul exemple que nous rencontrons, dans le gouvernement de Montréal, d'une diminution de territoire, lors d'un renouvellement de commission sous le régime militaire. Cette commission de Lalanne est datée du premier octobre 1760 .

Lalanne cessa de pratiquer en 1767.

Son greffe est déposé aux Archives judiciaires de Montréal.

\section{DOULLON Desmarets}

Le 20 février 1753, l'intendant Bigot accordait une commission de notaire royal dans les côtes de Pointe-Claire, Sainte-Anne du Bout de l'île, Lac des Deux-Montagnes, Châteauguay, et île Perrot seulement au sieur Doullon Desmarets. ${ }^{85}$

* Voir notre Revue, IX : 423-438, 546-561; X : 93-103, 257-263, 381-390; $\mathrm{XI}$ : 93-106.

$82 \mathrm{APQ}$, Ord. Int., XL: 5.

83 Ibid., XL: 57.

84 $R A C, 1918: 27 \mathrm{~s}$.

85 APQ, Ord. Int., XL: 55. 
Doullon Desmarets pratiquait également comme chirurgien. Il cessa d'exercer comme notaire en avril $1754 .^{86}$

\section{JEAN-BAPTISTE GUYART}

Voir l'inventaire des notaires royaux du gouvernement de Québec, vol. IX : 428 et suivantes.

\section{PIERRe PANeT}

Le 15 décembre 1754, Jean-Victor Varin, commissaireordonnateur pendant l'absence de Bigot, nommait Panet de Méru pour remplir la charge de notaire royal en la juridiction royale de Montréal, en remplacement du sieur Bouron, démissionnaire. ${ }^{87}$

Fait étrange, le 10 août 1756, on retrouve la même commission, cette fois de Bigot, donnée à Panet, en remplacement du sieur Bouron qui a démissionné. ${ }^{88}$

Le premier octobre 1760, Thomas Gage donnait une commission à Panet pour exercer l'office de notaire royal dans la ville de Montréal et dans toute l'étendue du gouvernement. ${ }^{89}$

Pierre Panet était le frère du notaire royal Jean-Claude Panet, qui exerçait à Québec. Sous le régime anglais, Pierre Panet devint avocat, continuant d'exercer comme notaire jusqu'en 1778. Il mourut juge à Montréal le 15 juin 1804. ${ }^{90}$

Le greffe de Pierre Panet de Méru est aux Archives judiciaires de Montréal.

\section{PhilipPe-Pierre Pilliamet}

Pilliamet, qui était suisse de nation, arriva au pays comme soldat. En 1748, il agissait comme sous-secrétaire du gouverneur de la Nouvelle-France. ${ }^{91}$

Le 15 avril 1755, "Estant Necessaire de remplir la place de Notaire En la jurisdiction Royale de cette ville Montréal vacante

$86 R A P Q, 1921-22$.

87 APQ, Ord. Int., XLI : 3.

88 Ibid., XLII : 20.

89 RAC, 1918: 25.

90 RAPQ, 1921-22: 57.

91 Ibid. 
par La mort du S. Adhemard qui En Estait cy devant pourvu », Varin nomme Pilliamet «pour faire les fonctions de Notaire Royal en L'Etenduë de La Jurisdiction de Cette ditte ville ${ }^{92}$

Le 16 août 1756, signée de Bigot, on retrouve exactement la même commission. ${ }^{93}$

Le greffe de Pilliamet est conservé aux Archives judiciaires de Montréal.

\section{ANTOINE GRISÉ}

L'intendant Bigot accordait, le 24 juillet 1756, une commission de notaire royal à Antoine Grisé pour exercer dans les seigneuries de Chambly et de Rouville. ${ }^{94}$

Le premier octobre 1760, Thomas Gage donnait une commission de notaire royal à Grisé, lui permettant d'exercer dans les paroisses de Chambly, Belœil et Rouville seulement, à la charge pour Grisé de demeurer à Chambly. ${ }^{95}$

Grisé exerça jusqu'en 1785, l'année de sa mort. ${ }^{96}$

Son greffe est aux Archives judiciaires de Montréal.

\section{PIERRE MeZIÈRE}

Pierre Mezière naquit en 1735 à Vilat, en Champagne. ${ }^{97}$

Le 25 mars 1758, Bigot lui accordait une commission de notaire royal en la juridiction de Montréal et dans tout le gouvernement de cette ville. ${ }^{98}$

Thomas Gage renouvelait la commission de Mezière, le premier octobre $1760 .{ }^{99}$

Mezière pratiqua jusqu'en $1786 .^{1}$

Son greffe est conservé aux Archives judiciaires de Montréal.

$92 \mathrm{APQ}$, Ord. Int., XLI: 8.

93 Ibid., XLII : 20.

94 Ibid., XLII : 17.

$95 R A C, 1918$ : 28.

$96 R A P Q, 1921-22$ : 57.

97 Tanguay, op. cit., VI : 19.

98 APQ, Ord. Int., XLII : 37s.

$99 R A C, 1918: 25 \mathrm{~s}$.

1 RAPQ, 1921-22: 58. 


\section{LOUIS DE COURVILLE}

Voir l'inventaire des notaires royaux du gouvernement de Québec, vol. IX: 428 et suivantes.

\section{PierRe Crevier DUVERnay}

Le dix-neuf juin 1762, Thomas Gage nommait Pierre Crevier Duvernay notaire royal dans toute l'étendue du gouvernement du Montréal, mais particulièrement dans les côtes de Verchères, Varennes et St-Ours, en remplacement de son père Jacques Crevier Duvernay, et à condition qu'il réside à Verchères. ${ }^{2}$

Pierre Crevier Duvernay devenait, avec Louis Joseph Soupras, nommé le même jour, le premier notaire royal du gouvernement de Montréal à être nommé sous le régime militaire. Jusqu'à eux, Gage n'avait fait que renouveler des commissions.

Crevier exerça jusqu'en 1801.

Le greffe de Pierre Crevier Duvernay est déposé aux Archives judiciaires de Montréal.

\section{LOUIS-JOSEPH SOUPRAS}

Louis-Joseph Soupras naquit à Montréal en $1730 .^{3}$

Le 19 juin 1762, il recevait de Thomas Gage une commission pour exercer l'office de notaire royal dans les paroisses de PointeClaire, Ste-Anne et Ste-Geneviève, à la charge de résider à Pointe-Claire. ${ }^{4}$

Soupras pratiqua jusqu'en 1792.

Son greffe est conservé aux Archives judiciaires de Montréal.

\section{LOUIS LOISEAU}

En remplacement d'Antoine Loiseau, Thomas Gage nommait, le 12 juillet 1762, Louis Loiseau Chalons fils notaire royal dans les paroisses de Boucherville et ses dépendances, à la charge pour Loiseau de résider à Boucherville. ${ }^{5}$

Son greffe est aux Archives judiciaires de Montréal.

$2 R A C, 1918: 57$.

3 Tanguay, op. cit., V: 184.

$4 R A C, 1918: 57$.

$5 R A C, 1918: 57 \mathrm{~s}$. 


\section{JEAN-MARIE ROULLET}

Jean-Marie Roullet du Châtellier était un ancien grenadier de la compagnie Foulhiac au régiment de Berry. ${ }^{6}$

Le 6 octobre 1762, il recevait de Thomas Gage une commission pour exercer comme notaire royal dans la paroisse de Varennes et ses dépendances, à la charge de demeurer à Varennes. $^{7}$

Roullet du Châtellier pratiqua jusqu'en 1781 .

Son greffe est déposé aux Archives judiciaires de Montréal.

\section{LOUIS-SIMON FRÉCHET}

Le 29 novembre 1762, Thomas Gage accordait une commission de notaire royal au sieur Louis Simon «Frichet» pour exercer dans les paroisses de St-Antoine, St-Charles, petit StOurs, Sorel et leurs dépendances, à la charge pour Fréchet de demeurer à St-Antoine. ${ }^{8}$

Fréchet exerça le notariat jusqu'en 1767.

Son greffe est aux Archives judiciaires de Sorel.

\section{BARTHELEMY FARIBAULT}

Barthelemy Faribault naquit dans le Mans, en 1713. Son père était huissier royal. Faribault arriva au pays en 1754 , et agit comme secrétaire de l'armée que commandait le Marquis Duquesne. Faribault décéda à Berthier en $1801 .^{9}$

Le 9 février 1763, Gage accordait à Faribault une commission de notaire royal dans les paroisses de Berthier, île Dupas, Lanoraie, Lavaltrie, St-Sulpice et leurs dépendances, à charge pour lui de demeurer à Berthier. ${ }^{10}$

Faribault pratiqua jusqu'à sa mort.

Le greffe de Faribault est déposé aux Archives judiciaires de Sorel.

6 J.-E. Roy, op. cit., II : 12.

$7 R A C, 1918: 61$.

8 Ibid., 64.

9 Tanguay, op. cit., IV: 9.

10 RAC, 1918: 68 . 


\section{FrançoIs RaCicot}

Le 20 juin 1763, François Racicot recevait une commission de notaire royal dans la paroisse de Boucherville et ses dépendances, en remplacement du sieur Chalons fils, démissionnaire, et à la charge de résider à Boucherville ${ }^{11}$

D'après Tanguay, ${ }^{12}$ Racicot serait né à Montréal en 1746. Il aurait donc été notaire à dix-sept ans, ce qui nous semble impossible. Il y a probablement erreur de Tanguay.

Racicot exerça jusqu'en 1796.

Son greffe est déposé aux Archives judiciaires de Montréal.

\section{II}

De 1664 à 1763 , quarante-six notaires royaux ont donc été appointés pour pratiquer à l'intérieur des limites du gouvernement de Montréal. De ce nombre, un ne pratiqua pas à Montréal (Jacques Barbel) et un autre (Jean de Latour) y exerça quelques semaines, par permission spéciale de l'intendant. $\mathbf{S i}$, au début du XVIII ${ }^{e}$ siècle, les notaires royaux étaient moins nombreux à Montréal qu'à Québec, on remarque qu'à la fin du régime français et sous le régime militaire ils dépassent en nombre ceux du gouvernement de Québec. Montréal prenait déjà figure de Métropole. Dans l'ensemble cependant, Québec compte un notaire royal de plus que Montréal.

Comme dans les autres gouvernements, il y eut à Montréal quelques notaires dont la qualité de notaires royaux reste douteuse. Suivant l'ordre que nous avons adopté jusqu'ici, nous ferons maintenant l'inventaire de ces notaires.

\section{PIERRE RAguideaU}

De Pierre Raguideau dit St-Germain, nous n'avons trouvé mention ni dans les listes officielles du notariat, ni dans l'Histoire $d u$ notariat de M. J.-E. Roy, ni même dans les Archives.

C'est dans un ouvrage de Mgr Tanguay, $A$ travers les registres, que nous avons rencontré le nom de Pierre Raguideau. A

11 Ibid., 72s.

12 Tanguay, op. cit., VI: 491. 
la date du 28 août 1665, Tanguay écrit: «Est inhumé le corps de Pierre Raguideau dit Saint-Germain, sergent royal de la Sénéchaussée de Montréal et notaire royal, victime de la cruauté des Iroquois. (Reg. de Montréal) ».13

Raguideau a-t-il réellement été notaire royal ? C'est un problème que nous n'avons pas encore résolu. Ce n'est pas impossible, quoiqu'il soit étonnant qu'on ne trouve nulle part mention de ce notaire. De toute façon, si Raguideau a pratiqué comme notaire royal, son greffe est aujourd'hui disparu.

\section{JACQUES BOURDON}

Jacques Bourdon était né à St-Godard, Rouen, en 1650.14

Il commença à exercer comme notaire seigneurial de Boucherville en 1677. En 1683, Pierre Boucher, seigneur de Boucherville, le révoquait et nommait à sa place Michel Moreau. ${ }^{15}$

Bourdon continua quand même de pratiquer jusqu'en 1720 , s'intitulant invariablement notaire royal. "Nous avons le droit de présumer qu'il avait reçu de l'intendant une commission de notaire royal $\gg .{ }^{16}$

Le greffe de Jacques Bourdon est conservé aux Archives judiciaires de Montréal. ${ }^{17}$

\section{JeAN-BAPTISTe Pottier}

Voir l'inventaire des notaires royaux du gouvernement des Trois-Rivières, IX : 258 et suivantes.

$$
\text { (à suivre) }
$$

\section{ANDRÉ VACHON}

13 Tanguay, A travers les registres, 48.

14 Tanguay, Dictionnaire généalogique, I : 78.

15 La lettre de révocation de Bourdon et de nomination de Moreau a été publiée dans Une vieille seigneurie, Boucherville, 401s.

$16 R A P Q, 1921-22$ : 27.

17 Voir Invent. des greffes des not., X: 69-97. 